\title{
Gauge Theories of the Strong and Electroweak Interaction
}

By Prof. Dr. rer. nat. Manfred Böhm, Universität Würzburg Dr. rer. nat. Ansgar Denner, Paul Scherrer Institut Villigen Prof. Dr. rer. nat. Hans Joos, DESY Hamburg 


\section{Contents}

1 Phenomenological basis of gauge theories of strong, electromagnetic, and weak interactions 1

1.1 Elementary particles and their interactions . . . . . . . . 1

1.1.1 Leptons and quarks as fundamental constituents of matter . . 2

1.1.2 Fundamental interactions . . . . . . . . . . . 4

1.2 Elements of relativistic quantum field theory . . . . . . . 5

1.2.1 Basic concepts of relativistic quantum field theory . . . . . 6

1.2.2 Lie algebras and Lie groups $\ldots \ldots \ldots \ldots \ldots \ldots$

1.2.3 Conserved currents and charges . . . . . . . . . . 24

1.3 The quark model of hadrons . . . . . . . . . . . . 29

1.3.1 Quantum numbers and wave functions of hadrons in the quark model . . . . . . . . . . . . . . . . . 29

1.3.2 Quark model with colour . . . . . . . . . . . . . 35

1.3.3 The concept of quark dynamics-quarkonia . . . . . . 36

1.4 Basics of the electroweak interaction . . . . . . . . . 39

1.4.1 Electroweak interaction of leptons . . . . . . . . . . . . 41

1.4.2 Electroweak interaction of hadrons . . . . . . . . . 47

1.5 The quark-parton model . . . . . . . . . . . . . 57

1.5.1 Scaling in deep-inelastic lepton-nucleon scattering . . . . . . 57

1.5.2 The parton model . . . . . . . . . . . . . . 63

1.5.3 Applications of the simple parton model $\ldots \ldots \ldots$. . . 68

1.5.4 Universality of the parton model . . . . . . . . . . . 72

1.6 Higher-order field-theoretical effects in QED . . . . . . . 76

1.6.1 QED as a quantum field theory $\ldots \ldots \ldots \ldots \ldots \ldots$ 
1.6.2 A test of QED: the magnetic moment of the muon . . . . . 78

1.7 Towards gauge theories of strong and electroweak interactions 81 References to Chapter $1 \ldots \ldots \ldots$. . . . . . 82

2 Quantum theory of Yang-Mills fields $\quad 85$

2.1 Green functions and $S$-matrix elements . . . . . . . . 86

2.1.1 The principles of quantum field theory $\ldots \ldots \ldots \ldots$. . 86

2.1 .2 Green functions . . . . . . . . . . . . . 88

2.1.3 S-matrix elements and the LSZ formula . . . . . . . . 95

2.1.4 Connected Green functions and vertex functions . . . . . . 97

2.1.5 Scattering of composite particles . . . . . . . . . . . 101

2.2 Path-integral representation of quantum field theory . . . . 104

2.2 .1 Functional calculus . . . . . . . . . . . . . . . . . 104

2.2.2 Generating functionals of Green functions $\ldots \ldots \ldots \ldots 111$

2.2.3 Functional-integral representation of the $S$ matrix . . . . . 115

2.2.4 The field-theoretical path integral . . . . . . . . . . 116

2.2.5 Feynman rules and path integral . . . . . . . . . . 122

2.2.6 Ward identities and equations of motion for Green functions . 127

$2.3 \quad$ Local gauge invariance . . . . . . . . . . . . . . 133

2.3.1 Local gauge invariance in QED . . . . . . . . . . . . . 134

2.3.2 Geometry of non-abelian gauge symmetry $\ldots \ldots \ldots \ldots 136$

2.3.3 Yang-Mills field theories . . . . . . . . . . . . . . 143

2.4 Path-integral formulation of gauge theories . . . . . . . . 148

2.4.1 Path-integral quantization of gauge theories . . . . . . . 149

2.4.2 Feynman rules for gauge theories . . . . . . . . . 157

2.4.3 BRS invariance and Slavnov-Taylor identities . . . . . . . 161

2.4.4 The background-field method . . . . . . . . . . 178

2.5 Renormalization of quantum field theories . . . . . . . 188

2.5.1 Divergences and renormalization . . . . . . . . . . . . 188

2.5.2 Calculation of one-loop corrections . . . . . . . . . 207

2.5.3 One-loop renormalization of gauge theories . . . . . . . . . 219 
2.5.4 Sketch of the proof of renormalizability of gauge theories . . 231

2.6 Renormalization group . . . . . . . . . . . . . . 252

2.6.1 Renormalization-group equation . . . . . . . . . . . 252

2.6.2 Renormalization-group function and anomalous dimensions of massless gauge theories . . . . . . . . . . . 260

2.6.3 Relation between different renormalization schemes . . . . . 265

2.6.4 Running unrenormalized coupling constant . . . . . . . . 267

$2.7 \quad$ Anomalies . . . . . . . . . . . . . . . 268

2.7 .1 The triangle-graph anomaly . . . . . . . . . . 269

2.7 .2 Anomalies in gauge theories $\ldots \ldots . \ldots . \ldots . \ldots 279$

2.8 Infrared and collinear singularities . . . . . . . . . 285

2.8.1 The origin of mass singularities . . . . . . . . . 286

2.8.2 Infrared singularities . . . . . . . . . . . . . . 294

2.8.3 Collinear singularities in QED . . . . . . . . . . . . . 305

2.9 Non-perturbative aspects of gauge theories . . . . . . . . 328

2.9.1 Topological quantum numbers . . . . . . . . . . . . . . 329

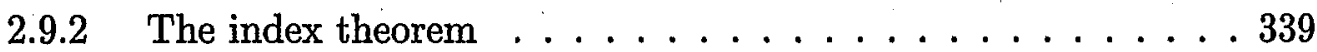

2.9.3 Path integral and topology . . . . . . . . . . . . 347

2.10 Lattice approximation of gauge theories . . . . . . . . . . 379

2.10.1 Basics of lattice approximation . . . . . . . . . . . . 379

2.10 .2 Strong-coupling approximation . . . . . . . . . . . 385

2.10 .3 Numerical methods . . . . . . . . . . . . . . . . 390

2.10.4 Transition to the continuum . . . . . . . . . . . . . 397

2.10.5 Finite-size effects . . . . . . . . . . . . . . . . 404

2.10.6 Lattice approximation of fermionic interactions . . . . . . 406 References to Chapter $2 \ldots \ldots \ldots . \ldots . \ldots 415$ 
3 Quantum Chromodynamics $\quad 426$

3.1 Asymptotic freedom of QCD . . . . . . . . . . . . 427

3.1.1 The running coupling constant of QCD . . . . . . . . . 427

3.1.2 Importance of higher-order corrections . . . . . . . . 430

3.1.3 The running quark masses of QCD . . . . . . . . . . . 432

3.2 QCD in deep-inelastic scattering . . . . . . . . . . 435

3.2.1 The field-theoretical approach to the parton model . . . . 436

3.2.2 QCD corrections to the parton model . . . . . . . . . . 447

3.2.3 Evolution equations . . . . . . . . . . . . . . 458

3.2.4 Experimental tests of QCD . . . . . . . . . . . . . 464

3.3 Perturbative Quantum Chromodynamics . . . . . . . . 469

3.3.1 One-loop corrections to the parton model . . . . . . . . . . 470

3.3 .2 Factorization .................. 476

3.3.3 Factorization and the operator-product expansion . . . . . 479

3.3.4 Lepton-pair production in hadron-hadron scattering . . . . 484

3.3.5 Jet cross sections . . . . . . . . . . . . . . . . 488

3.3.6 Total hadronic cross section . . . . . . . . . . . . . 494

3.4 Heavy-quark effective theory (HQET) . . . . . . . . . . 497

3.4.1 The Lagrangian of HQET . . . . . . . . . . . . . . 498

3.4.2 Symmetries of HQET . . . . . . . . . . . . . . . 502

3.4.3 Applications of HQET . . . . . . . . . . . . . . . . 504

3.5 Light quarks and chiral perturbation theory . . . . . . . . 507.

3.5.1 Chiral symmetry of massless QCD . . . . . . . . . . . . 507

3.5.2 Pion-pole dominance and effective low-energy theory . . . . 510

3.5.3 The non-linear $\sigma$ model . . . . . . . . . . . . . . . . 512

3.5.4 Breaking of chiral invariance . . . . . . . . . . . 513

3.5.5 Applications of chiral perturbation theory . . . . . . . . 516

3.6 Results of lattice approximation of QCD . . . . . . . . 518

3.6.1 The hadron spectrum . . . . . . . . . . . . . . . 519

3.6 .2 Glue balls ... . . . . . . . . . . . . . . . 524 
3.6.3 The connection between long- and short-distance physics: nonperturbative renormalization group . . . . . . . . 5 526

3.7 Quark confinement . . . . . . . . . . . . . . . . 529

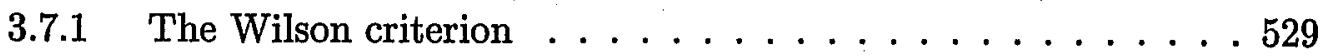

3.7.2 Quark confinement in strong-coupling approximation . . . . .534

3.7 .3 The string picture . . . . . . . . . . . . . . 536

3.7.4 Long-range correlations of the QCD vacuum . . . . . . . . . 548

$3.8 \quad$ A test of QCD: the running of $\alpha_{\mathrm{s}} \ldots \ldots \ldots \ldots 54$ References to Chapter $3 \ldots \ldots \ldots 57$

4. Gauge theories of the electroweak interaction $\quad 566$

4.1 Spontaneous symmetry breaking . . . . . . . . . . . 5667

4.1.1 Spontaneous breaking of a global symmetry . . . . . . . 568

4.1.2 Spontaneous breaking of a gauge symmetry . . . . . . 580

4.2 The Standard Model of the Electroweak Interaction . . . . . . 591

4.2.1 The Lagrangian of the Electroweak Standard Model . . . . . 592

4.2.2 The Lagrangian in the physical basis . . . . . . . . . . 596

4.3 Simple applications of the Electroweak Standard Model . . . . 604

4.3.1 W-pair production in $\mathrm{e}^{+} \mathrm{e}^{-}$annihilation . . . . . . . . . . .604

4.3.2 Production and decay of the Higgs boson . . . . . . . . 613

4.4 Quantization of the Electroweak Standard Model . . . . . . 622

4.4.1 Gauge fixing and Faddeev-Popov fields . . . . . . . . . . 622

4.4.2 BRS symmetry and physical fields . . . . . . . . . . . 625

4.4 .3 Slavnov-Taylor identities . . . . . . . . . . . . . . . . 628

4.4 .4 Lee identities . . . . . . . . . . . . . . . . 630

4.4.5 The background-field method for the Electroweak Standard Model

4.4.6 Charge universality in the Electroweak Standard Model . . . 642

4.4.7 The Goldstone-boson equivalence theorem . . . . . . . . . . 646

4.5 Renormalization of the Electroweak Standard Model . . . . 651

4.5.1 The renormalization transformation in the on-shell scheme . . 652 
X Contents

4.5.2 Renormalization conditions . . . . . . . . . 655

4.5.3 Explicit form of renormalization constants . . . . . . . . 661

4.5.4 Renormalization within the background-field method . . . . 666

4.5.5 Mass renormalization for unstable particles . . . . . . . . 668

4.6 Electroweak radiative corrections . . . . . . . . . 671

4.6.1 Fermionic contributions to the gauge-boson self-energies $\ldots 672$

4.6.2 Parameter relations in higher orders . . . . . . . . . . 675

4.6.3 Decay widths of the weak gauge bosons . . . . . . . . . . 684

4.6.4 Z-boson physics . . . . . . . . . . . . . . . 689

4.6.5 Precision tests of the electroweak interaction . . . . . . . 704

4.6.6 Status of the Electroweak Standard Model and perspectives . 706

References to Chapter 4 . . . . . . . . . . . . . . 709

$5 \quad$ Extensions of the Standard Model $\quad 716$

5.1 Grand unified theories (GUTs) . . . . . . . . . . . 717

5.1.1 Unification of coupling constants . . . . . . . . . .718

5.1 .2 Proton decay . . . . . . . . . . . . . . . . . 721

5.1.3 Hierarchy (fine-tuning) problem . . . . . . . . . . . 723

$5.1 .4 \quad \mathrm{SU}(5) \mathrm{GUT} \ldots \ldots \ldots \ldots . \ldots \ldots . \ldots \ldots$

$5.2 \quad$ Supersymmetry . . . . . . . . . . . . . . . . . 732

5.2 .1 Supersymmetry algebra . . . . . . . . . . . . . . 732

5.2.2 The chiral multiplet and the Wess-Zumino model . . . . . 733

5.2.3 Improved ultraviolet properties of supersymmetric theories . . 734

5.2.4 The Minimal Supersymmetric Standard Model (MSSM) . . 736

5.2.5 Supersymmetric grand unification . . . . . . . . . . 738

References to Chapter $5 \ldots \ldots$. . . . . . . . 740 
$\begin{array}{lll}\text { A Appendix } & \mathbf{7 4 2}\end{array}$

A.1 Conventions . . . . . . . . . . . . . . . . 742

A.1.1 Minkowski space. . . . . . . . . . . . . . . 742

A.1.2 Euclidean space . . . . . . . . . . . . . 750

A.1.3 Field theory . . . . . . . . . . . . . . 751

A.2 . Feynman rules for the Electroweak Standard Model . . . . . 758

$\begin{array}{ll}\text { General references } & \mathbf{7 6 5}\end{array}$

$\begin{array}{ll}\text { Index } & \mathbf{7 6 6}\end{array}$

\section{Corrections to this book}

A list of misprints and corrections to this book is posted on the WorldWide Web at the URL http://ww. hep.psi.ch/denner/GTupdates.html, or can be obtained by writing to the authors. We would be grateful, if you would report addtional errors in the book to Ansgar.Denner@psi.ch. 